\title{
Multiple myeloma with a previous diagnosis of focal segmental glomerulosclerosis: A case report and review of the literature
}

\author{
ZHE-YI DONG $^{1,2}$, HAI-TAO XING ${ }^{1,3}$, YUAN-DA WANG $^{1}$, WEI ZHANG ${ }^{1}$, QIANG QIU ${ }^{1}$ and XIANG-MEI CHEN ${ }^{1}$ \\ ${ }^{1}$ Department of Nephrology, State Key Laboratory of Kidney Disease, National Clinical Research Center for Kidney Disease, \\ Chinese People's Liberation Army General Hospital, Beijing 100853; ${ }^{2}$ Department of Nephrology, \\ The 309 Hospital of the Chinese People's Liberation Army, Beijing 100091; ${ }^{3}$ Department of Nephrology, \\ First Affiliated Hospital of Tianjin University of Traditional Chinese Medicine, Tianjin 300193, P.R. China
}

Received September 18, 2014; Accepted July 28, 2015

DOI: $10.3892 / \mathrm{ol} .2015 .3669$

\begin{abstract}
The presentation of focal segmental glomerulosclerosis (FSGS) and multiple myeloma (MM), either together or in succession, is extremely rare. Only nine studies have previously reported this poorly understood association. The present study reports the case of a 45-year-old male with FSGS that was diagnosed by a renal biopsy performed for nephrotic syndrome (NS). The patient was admitted to the Chinese People's Liberation Army General Hospital one year later with a fever, anemia, unresolved NS and renal insufficiency. The patient was diagnosed with MM and a renal biopsy was repeated, the results of which suggested renal amyloidosis. The MM was treated with three cycles of vincristine, doxorubicin and dexamethasone chemotherapy. A review of the literature indicated that monoclonal gammopathy may lead to FSGS. It suggested that FSGS patients who are $>40$ years old should be routinely screened for plasma cell proliferative disorders to guide the treatment, determine a prognosis, achieve primary disease remission and avoid end-stage renal disease.
\end{abstract}

\section{Introduction}

Plasma cell proliferative disorders are characterized by clonal proliferation of plasma cells, which produce either entire immunoglobulins (Igs) or Ig fragments (1). Monoclonal gammopathy of undetermined significance (MGUS)

Correspondence to: Dr Qiang Qiu or Dr Xiang-Mei Chen, Department of Nephrology, State Key Laboratory of Kidney Disease, National Clinical Research Center for Kidney Disease, Chinese People's Liberation Army General Hospital, 28 Fuxing Road, Beijing 100853, P.R. China

E-mail: medsage@126.com

E-mail: zheyidong@gmail.com

Key words: focal segmental glomerulosclerosis, plasma cell proliferative disorders, multiple myeloma, monoclonal gammopathy of undetermined significance and multiple myeloma ( $\mathrm{MM}$ ) are the most common plasma cell proliferative disorders (2). Approximately $1 \%$ of adults $>50$ years old have MGUS, with a 14-fold risk of developing MM. In 2012, there were 11,425 new patients with myeloma globally, and it is predicted that MM will be diagnosed in over 2,000 patients in the United States alone in 2015 (3). It is currently unknown whether the occurrence of focal segmental glomerulosclerosis (FSGS) in these patients is associated with the primary disease (1). Therapy for secondary FSGS should be directed at the underlying disorder, and corticosteroids are the mainstay of treatment for idiopathic FSGS (4). Furthermore, following a retrospective review of FSGS and MM English-language studies, nine publications were identified. Of these studies, seven demonstrated an association between FSGS and plasma cell proliferative disorders (2,5-10). However, the other two studies indicated little or no correlation between the two conditions $(11,12)$. As FSGS and MM occur infrequently in the same patient and there are few relevant previous studies, the association between them remains unclear. The current study describes a confirmed case of MM in a patient diagnosed with FSGS and renal amyloidosis on two successive renal biopsies. According to the literature, a correlation between the two disorders may be present. Written informed consent was obtained from the patient for the publication of this case report.

\section{Case report}

In April 2012, a 45-year-old male patient presented to the Chinese People's Liberation Army General Hospital (Beijing, China) with intermittent edema in the lower extremities, fever and fatigue. The patient had previously been diagnosed with nephrotic syndrome (NS) one year earlier at China-Japan Friendship Hospital (Beijing, China). The pathology report on the renal biopsy performed in the previous hospital indicated tip variant FSGS (Fig. 1). Following treatment with glucocorticoids and tripterygium glycosides for one year (Table I), the patient's urine protein levels decreased, but the serum creatinine levels increased. Additionally, the patient's blood pressure and hemoglobin levels progressively decreased (Table II). Physical examination revealed the patient had a temperature of $37^{\circ} \mathrm{C}$ (normal 
Table I. Continuous treatments in the previous hospital.

Treatment at indicated times

\begin{tabular}{|c|c|c|c|c|c|}
\hline Drug & April 2012 & November 2012 & December 2012 & March 2013 & June 2013 \\
\hline Methylprednisolone & $40 \mathrm{mg}$ i.v. 3D & & & & \\
\hline Prednisone & 60 mg q.d. & 30 mg q.d. & 10 mg q.d. & 25 mg q.d. & 10 mg q.d. \\
\hline TGT & & $20 \mathrm{mg}$ tid & $40 \mathrm{mg}$ tid & $20 \mathrm{mg}$ tid & $20 \mathrm{mg}$ tid \\
\hline Leflunomide & & & & & $10 \mathrm{mg}$ q.d. \\
\hline
\end{tabular}

TGT, tripterygium glycosides tablets; i.v., intravenous injection; 3D, daily for 3 days; q.d., every day; tid, three times per day.

Table II. Blood pressure and laboratory test results from the previous hospital treatment.

\begin{tabular}{|c|c|c|c|c|c|c|}
\hline \multirow[b]{2}{*}{ Parameter } & \multicolumn{6}{|c|}{ Values at indicated times } \\
\hline & April 2012 & November 2012 & December 2012 & March 2013 & June 2013 & July 2013 \\
\hline $\mathrm{SBP}, \mathrm{mmHg}$ & 130 & 111 & 127 & 107 & 87 & 85 \\
\hline $\mathrm{DBP}, \mathrm{mmHg}$ & 80 & 78 & 72 & 67 & 59 & 55 \\
\hline $\mathrm{MBP}, \mathrm{mmHg}$ & 123 & 115 & 114 & 103 & 88 & 83 \\
\hline 24-h urine protein, $\mathrm{g}$ & 21.03 & 5.15 & 6.66 & 9.57 & 9.45 & 7.53 \\
\hline WBC, $1 \times 10^{9}$ cells $/ 1$ & 8.65 & 13.5 & 15.9 & 14.3 & 12 & 8.97 \\
\hline $\mathrm{HGB}, \mathrm{g} / \mathrm{l}$ & 111 & 147 & 138 & 127 & 82 & 72 \\
\hline ALB, g/l & 16.4 & 23.1 & 22.8 & 20.4 & 23.3 & 15.4 \\
\hline $\mathrm{Scr}, \mu \mathrm{mol} / 1$ & 70 & 64.52 & 68.07 & 69.84 & 91.05 & 100 \\
\hline BUN, mmol/l & 5.65 & 6.65 & 6.76 & 5.72 & 5.83 & 5.26 \\
\hline $\mathrm{UA}, \mu \mathrm{mol} / 1$ & 366 & 395 & 464 & 514 & 544 & 419 \\
\hline $\mathrm{GFR}, \mathrm{ml} / \mathrm{min} / 1.73 \mathrm{~m}^{2}$ & 80.70 & 94.96 & 89.38 & 86.17 & 68.75 & 55.84 \\
\hline
\end{tabular}

SBP, systolic blood pressure; DBP, diastolic blood pressure; MBP, mean arterial pressure; WBC, white blood cell count; HGB, hemoglobin; ALB, albumin; Scr, serum creatine; BUN, blood urea nitrogen; UA, uric acid; GFR, glomerular filtration rate.

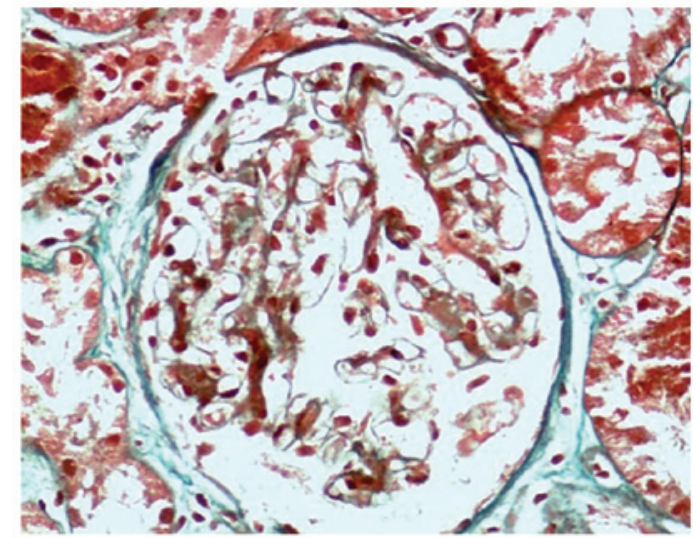

Figure 1. Masson trichome staining of kidney tissue (original magnification, $\mathrm{x} 400$ ).

range, $36-37^{\circ} \mathrm{C}$ ) and a body-mass index of $26.1 \mathrm{~kg} / \mathrm{m}^{2}$ (normal range, $18.5-22.9 \mathrm{~kg} / \mathrm{m}^{2}$ ).

Laboratory examinations revealed a calcium level of $2.19 \mathrm{mmol} / 1$ (normal range, 2.09-2.54 $\mathrm{mmol} / \mathrm{l}$ ). The serum immunoglobulin $\mathrm{G}$ (IgG) level was 2,950.0 mg/dl (normal range,
$700-1,600 \mathrm{mg} / \mathrm{dl}$ ), the $\kappa$-light chain level was $16.6 \mathrm{mg} / \mathrm{dl}$ (normal range, $170-370 \mathrm{mg} / \mathrm{dl}$ ) and the $\lambda$-light chain level was $810.0 \mathrm{mg} / \mathrm{dl}$ (normal range, 90-210 mg/dl). Serum immunofixation electrophoresis revealed IgG $\lambda$ MGUS. The patient's urinary $\kappa$-light chain level was $1.36 \mathrm{mg} / \mathrm{dl}$ (normal range, $0-0.79 \mathrm{mg} / \mathrm{dl}$ ) and the $\lambda$-light chain level was $135.0 \mathrm{mg} / \mathrm{dl}$ (normal range, $0-0.43 \mathrm{mg} / \mathrm{dl}$ ). Ultrasound of the renal artery indicated an increased resistance index for the initial segment, and lesions in the renal microvasculature. Ultrasound revealed that the left and right kidneys measured $12.9 \times 7.8 \times 6.4 \mathrm{~cm}$ and $13.7 \times 7.0 \times 5.7 \mathrm{~cm}$, respectively (normal range, 10.0-12.0x5.0-6.0x3.0-4.0 cm). A bone marrow biopsy revealed that $36 \%$ of the plasma cells were abnormal and a diagnosis of $\operatorname{IgG} \lambda$-type MM was formed.

Renal biopsy. Periodic acid-Schiff (PAS) staining revealed 16 intact glomeruli with normal volumes. Although there was global sclerosis in one glomerulus $(6.3 \%)$, there was no glomerular adhesion to the Bowman's capsule, segmental sclerosis or glomerular crescent. Furthermore, there was diffuse mild-to-moderate mesangial broadening, but no mesangial cell proliferation. The PAS staining was light in the mesangial area and there was a deposit of a homogeneous, protein-like substance, which was also observed in the renal interstitium 


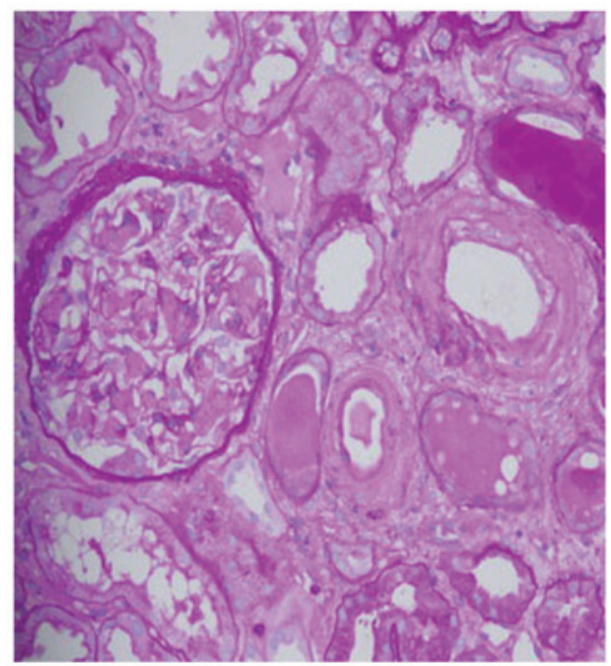

Figure 2. Deposition of a lightly stained, homogeneous, protein-like substance observed in arterial walls and glomerular mesangial areas (periodic acid-Schiff staining; original magnification, x200).

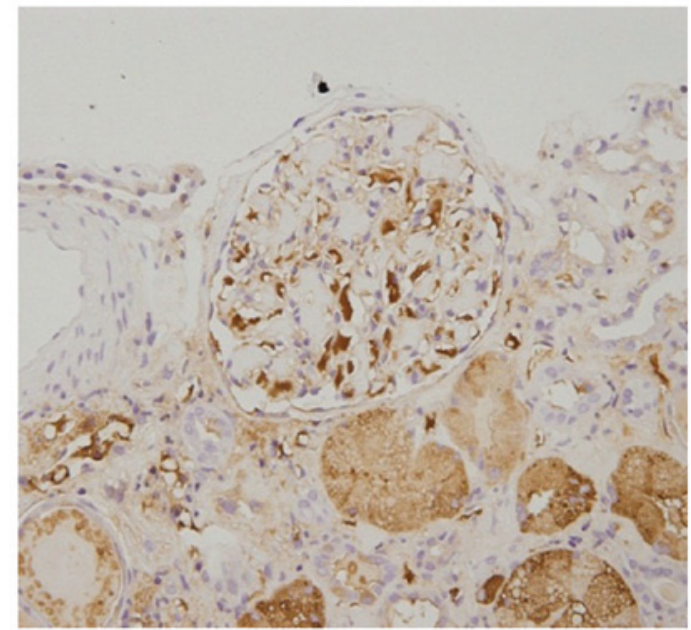

Figure 3. Glomerular and tubular epithelial cells positive for $\lambda$-light chains, identified by immunohistochemistry (original magnification, $\mathrm{x} 400$ ).

and on the walls of the renal artery. In addition, a number of capillary loops exhibited restricted openings (Fig. 2). The renal tubules were diffuse and severely atrophied, and protein casts were visible. A few plasma cells were observed to be diffused through interstitial spaces. Immunohistochemistry demonstrated that the glomerular and tubular epithelial cells were $\lambda$ chain-positive and $\kappa$ chain-negative (Figs. 3 and 4). Electron microscopy revealed collagen fiber hyperplasia (Figs. 5 and 6).

Treatment and outcomes. Following discharge, the patient returned to a local hospital to receive chemotherapy for the MM. The patient's oncologist commenced treatment for MM with vincristine, doxorubicin and dexamethasone. Following three cycles of drug treatment, the NS was resolved and renal function returned to normal. Upon follow-up examination, the patient's IgG level was $1,480 \mathrm{mg} / \mathrm{dl}$, the $\kappa$-light chain level was $316 \mathrm{mg} / \mathrm{dl}$ and the $\lambda$-light chain was $91 \mathrm{mg} / \mathrm{dl}$. No antibiotics were administered and the patient's body temperature returned to a normal level. The patient has since been lost to follow-up.

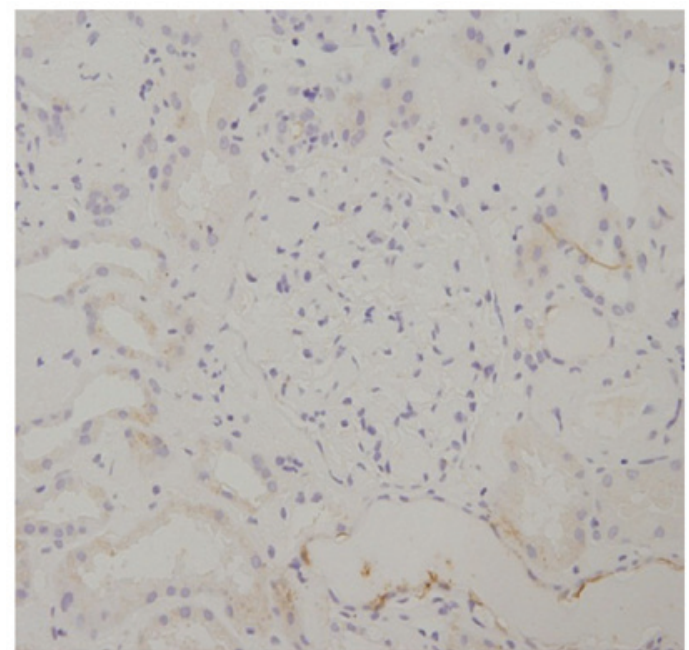

Figure 4. Glomerular and tubular epithelial cells negative for $\kappa$-light chains, identified by immunohistochemistry (original magnification, $\mathrm{x} 400$ ).

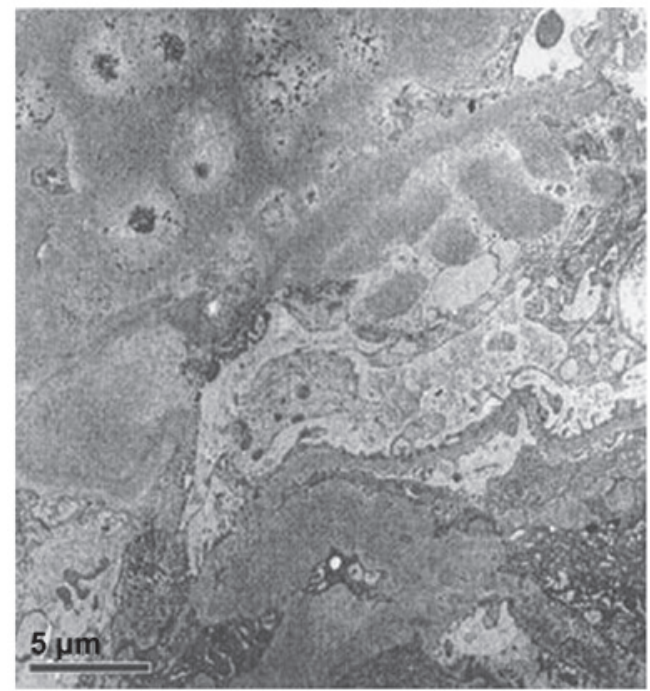

Figure 5. Electron micrograph demonstrating no cellular broadening or randomly distributed fibers in the glomerular basement membrane and mesangial areas.

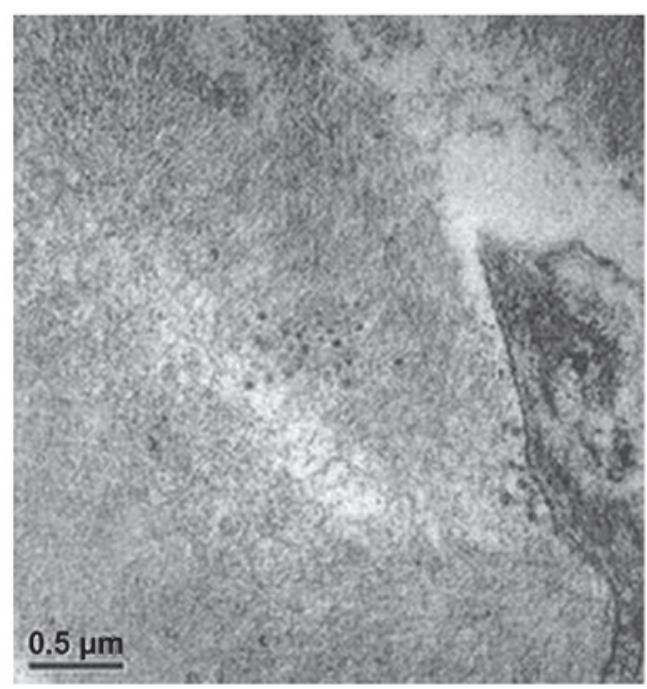

Figure 6. Electron micrograph demonstrating collagen fiber hyperplasia and randomly distributed fibrous tissues in the renal interstitium. 


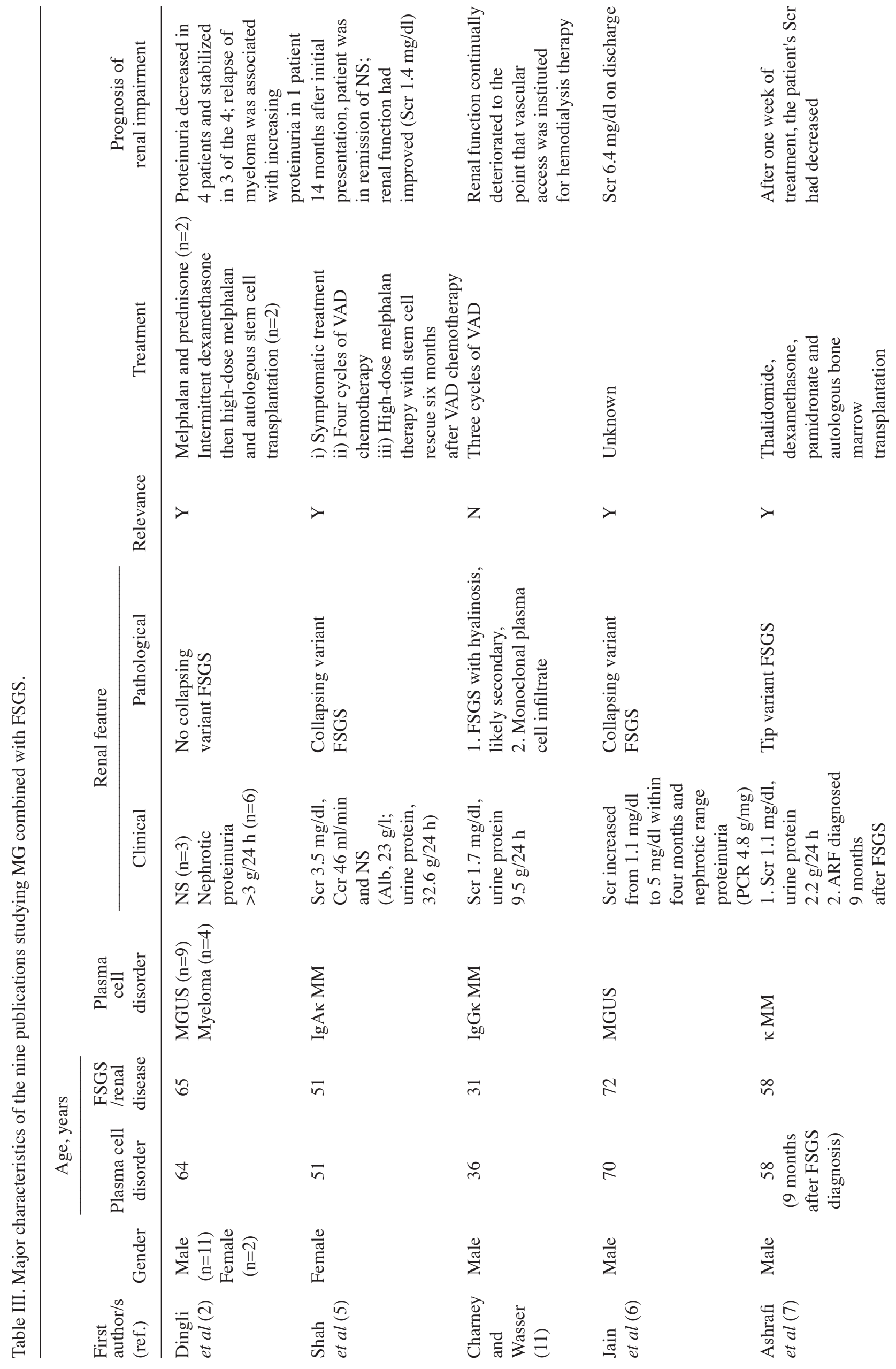



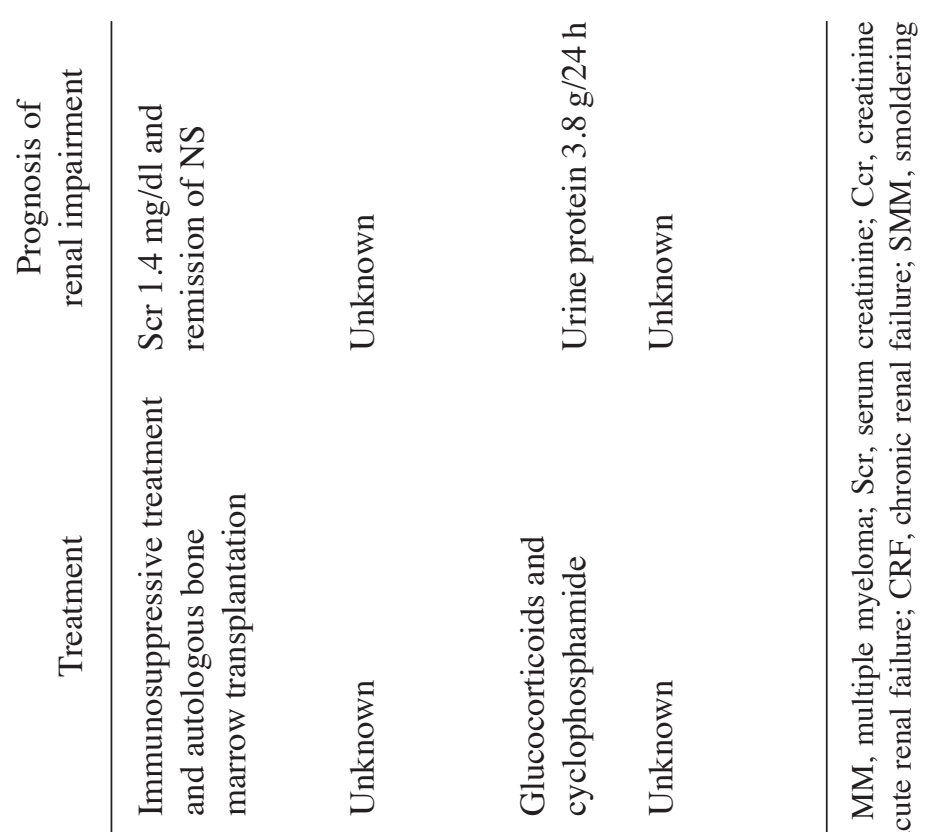

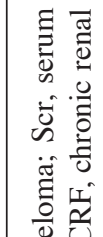
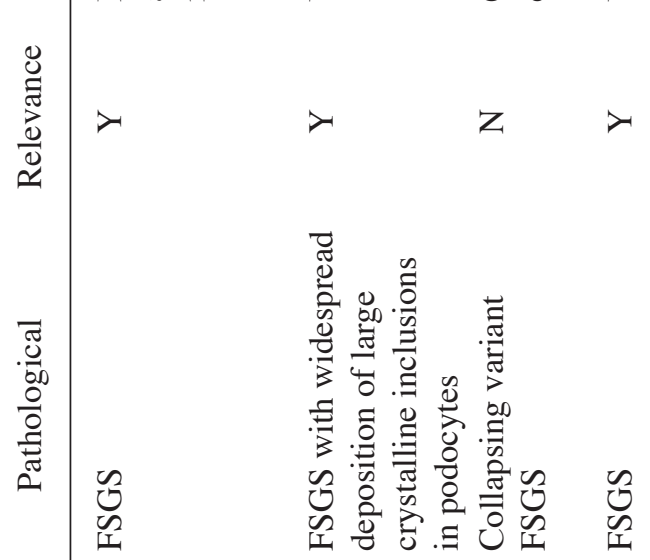

뜰
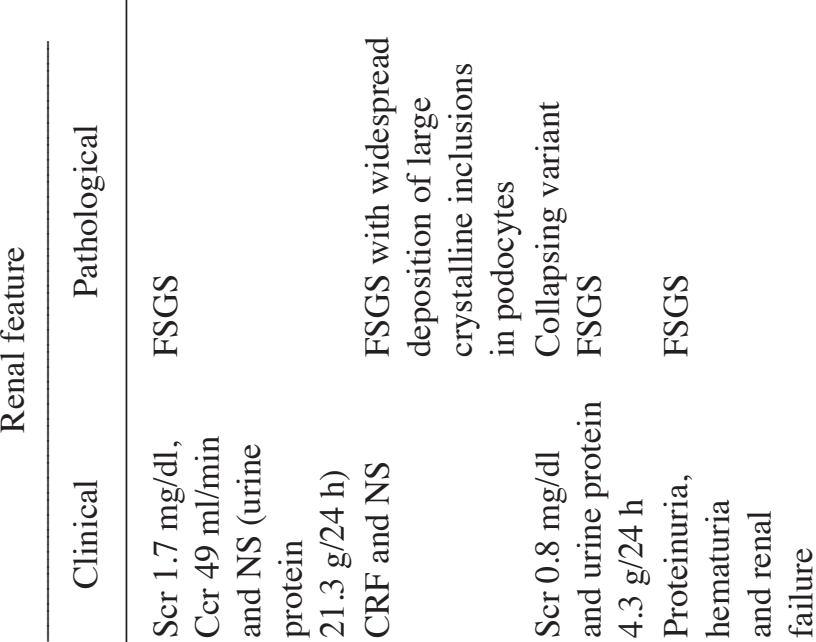

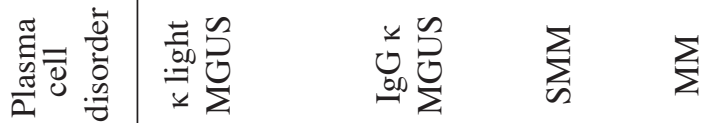




\section{Discussion}

FSGS is divided into primary and secondary subtypes, and there are several known causes of the latter. FSGS secondary to the hematological tumors T-cell lymphoma (13) or Hodgkin's lymphoma (14) are the most common and their associations are clear. FSGS secondary to a large granular lymphocytosis (15) is less common, but their association has also been documented. Cases of FSGS with MM or MGUS (2) are rare and the link between them is unclear and speculative.

The patient described in the current study was pathologically diagnosed with FSGS one year prior to admission. However, the NS did not resolve following first-line treatment and the patient's renal function declined. Several phenomena associated with MM were noted, such as anemia that did not parallel the decline in renal function, a reduction in blood pressure that was associated with drug utilization and an increased monoclonal immunoglobulin level in the serum. Therefore, while forming the diagnosis of MM via bone marrow biopsy, a repeat renal biopsy was performed on the patient, which confirmed renal amyloidosis.

Valizadeh et al (10) reported the case of a patient who had been pathological diagnosed with FSGS and was confirmed with MM upon follow-up. The study speculated that MM may be a rare secondary occurrence to FSGS. Although the present patient was diagnosed with renal amyloidosis after MM, a missed diagnosis of FSGS-like lesions as a result of not performing serial pathological sections cannot be excluded. FSGS is a morphological diagnostic term. Secondary FSGS can be a morphological change in a variety of diseases that develop to a certain stage rather than being caused by a single disease. Secondary FSGS has relatively clear risk factors and FSGS-like changes are usually present during the development of primary glomerular diseases (4). Electron microscopy (EM) is currently, the best method for identifying FSGS, and primary FSGS is highly suggested if it indicates the disappearance of $>80 \%$ of diffuse foot processes (16). However, EM for the present study indicated renal amyloidosis without changes in the foot processes. On this basis, the possibility of MM combined with primary FSGS was excluded.

The patient's reduced blood pressure indicated a decline in vascular elasticity and suggested the deposition of amyloid substances on the vascular wall. Ultrasound of the renal artery demonstrated an increased resistance index of the initial segment. This may have been due to amyloid substances on the walls of the renal microvasculature narrowing the luminal spaces or to the deposition of amyloid substances in the mesangial area that may have restricted the openings of the capillary loops. Furthermore, the renal pathology results confirmed a similar inference. We hypothesize that restricted openings of certain capillary loops are compensated for by the capillary loops with unobstructed openings, and increased pressure and perfusion inside the glomeruli are inevitable. This leads to secondary FSGS by compensatory changes, which negatively affect the structure and function of the glomeruli. Thus, from the pathophysiological perspective, the renal pathology of patients may indeed demonstrate FSGS. However, the study by Valizadeh et al (10) did not repeat the renal biopsy following the patient's MM diagnosis and did not perform Congo red staining or light chain immunological testing on the original pathological sections. Therefore, the pathological changes in the kidney could not be confirmed following the MM diagnosis.

Studies on the association between monoclonal gammopathies and FSGS are rare. Only nine publications were identified in a retrospective review of the English-language literature (Table III). Of these studies, three suggested that there was little or no correlation between FSGS and plasma cell proliferative disorders. In a study by Shah et al (12), the NS of the patient was not resolved by hormone therapy, however, the patient's smoldering MM did improve. Paueksakon et al (1) identified that 13 out of 87 (14.9\%) patients with MGUS and renal damage had FSGS-like lesions, and thus, the FSGS was not considered to be primary. Charney and Wasser (11) demonstrated in their study population that obesity and sleep apnea were more relevant to FSGS. These studies did not identify a correlation between these two diseases, as there was no evidence of MM-induced renal damage, such as amyloidosis, cast nephropathy or plasma cell infiltration. However, a secondary FSGS diagnosis does not require renal damage from the primary disease. In addition, the treatment of MM in the study by Charney and Wasser (11) was not continuous, remission rates were poor and renal failure also progressed to end-stage renal disease. All of these factors support the notion that the two diseases are associated. While the patients in a study by Shah et al (12) exhibited a reduction in serum monoclonal protein and light chain protein levels following the use of hormones for 3 months, the proteinuria did not resolve. It is possible that the improvement in secondary FSGS required a longer time than that required to control the primary disease. Treatment was subsequently commenced with cyclophosphamide, which increased the remission rate for MM compared to cyclosporine, and improved the FSGS. The improved therapeutic effect of cyclophosphamide on FSGS during MM supports the association between FSGS and MM. The correlation between FSGS and MM becomes clearer when patients use pamidronate. Treatment of osteolytic complications in MM patients using pamidronate can result in the collapsing variant of FSGS (17). The simultaneous occurrence of FSGS and $\mathrm{MM}$ is not a coincidence; Dingli et al (2) reported the cases of 13 patients who were diagnosed with syndromes secondary to plasma cell proliferative disorders (four cases of $\mathrm{MM}$ and nine cases of MGUS). For all 13 cases, renal damage improved following treatment of the primary diseases and recurred with the relapse of the primary diseases. Therefore, a common pathogenic link between MGUS and MM is plausible.

Vascular endothelial growth factor (VEGF) and heparanase can change the glomerular permeability and affect the glomerular basement membrane function to cause proteinuria in FSGS (18). VEGF and heparanase are overexpressed in MM patients, and VEGF can induce the growth of bone marrow blood vessels $(19,20)$ Heparanase plays important roles in tumor growth, angiogenesis and metabolism. Furthermore, these two proteins are involved in the occurrence and development of MGUS and MM (19,20). Dingli et al (2) used a causal inference epidemiological method to note the close association between MGUS, MM and FSGS.

The present study indicates that FSGS patients $>40$ years old should undergo serum and urinary immune 
electrophoresis, Congo red staining, and $\kappa$ - and $\lambda$-light chain level analysis routinely to detect plasma cell proliferative disorders and improve the prognosis of potentially associated FSGS. Additionally, repeated renal pathological examinations may be required for a small fraction of patients.

\section{Acknowledgements}

This study was supported by the pathologists of the State Key Laboratory of Kidney Disease in the Chinese People's Liberation Army General Hospital. Additionally, this study was partially supported by the Army Medical Science Youth Development Project (grant no. 13QNP180) and the Project of Chinese PLA 309 Hospital (grant no. 2014MS-006). The authors would like to thank Professor Zhang Xue-Guang and Mr. Yin Zhong for sharing their pathological materials.

\section{References}

1. Paueksakon P, Revelo MP, Horn RG, Shappell S and Fogo AB Monoclonal gammopathy: Significance and possible causality in renal disease. Am J Kidney Dis 42: 87-95, 2003.

2. Dingli D, Larson DR, Plevak MF, Grande JP and Kyle RA: Focal and segmental glomerulosclerosis and plasma cell proliferative disorders. Am J Kidney Dis 46: 278-282, 2005.

3. Gertz MA: Utility of the immunoglobulin free light chain assay for plasma cell disorders 2015. Leuk Lymphoma: March 27, 2015 (Epub ahead of print).

4. Deegens JK, Steenbergen EJ and Wetzels JF: Review on diagnosis and treatment of focal segmental glomerulosclerosis. Neth J Med 66: 3-12, 2008

5. Shah S, Cavenagh J, Sheaf M and Thuraisingham RC: Remission of collapsing focal segmental glomerulosclerosis following chemotherapy for myeloma. Am J Kidney Dis 43: e10-e12, 2004.

6. Jain N, Tantaco MR, Thomas B and Laut J: Monoclonal gammopathy of uncertain significance (MGUS) and focal segmental glomerulosclerosis (FSGS) - A case report. Am J Kidney Dis 55: B56, 2010.

7. Ashrafi F, Mortazavi M, Manouchehri N, Moghaddam NA, Nasri H and Sarrami AH: Focal segmental glomerulosclerosis, secondary amyloidosis and multiple myeloma. Pak J Med Sci 28 : 345-347, 2012.
8. Torun D, Canpolat T and Özelsancak R: Focal Segmental Glomerulosclerosis in a patient associated with kappa-light chain disease. Turk Neph Dial Transpl 20: 96-98, 2011.

9. Matsuyama N, Joh K, Yamaguchi Y, et al: Crystalline inclusions in the glomerular podocytes in a patient with benign monoclonal gammopathy and focal segmental glomerulosclerosis. Am J Kidney Dis 23: 859-865, 1994.

10. Valizadeh N, Makhdomi K, Noroozinia F and Behzadi F. Multiple myeloma presenting with focal segmental glomerulosclerosis. South Asian J Cancer 2: 168, 2013.

11. Charney DA and Wasser W: A 36-year-old man with a monoclonal gammopathy and nephrotic syndrome. Am J Kidney Dis 42: 1097-1101, 2003.

12. Shah R, Shah N, Shah A and Mehta AN: Steroid-resistant nephrotic syndrome secondary to primary focal segmental glomerulosclerosis and smoldering multiple myeloma. Proc (Bayl Univ Med Cent) 27: 19-21, 2014.

13. Belghiti D, Vernant JP, Hirbec G, Gubler MC, Andre C and Sobel A: Nephrotic syndrome associated with T-cell lymphoma. Cancer 47: 1878-1882, 1981.

14. Hyman LR, Burkholder PM, Joo PA and Segar WE: Malignant lymphoma and nephrotic syndrome. A clinicopathologic analysis with light, immunofluorescence, and electron microscopy of the renal lesions. J Pediatr 82: 207-212, 1973.

15. Bassan R, Rambaldi A, Abbate M, et al: Association of NK-cell lymphoproliferative disease and nephrotic syndrome. Am J Clin Pathol 94: 334-338, 1990.

16. Bose B and Cattran D; Toronto Glomerulonephritis Registry: Glomerular diseases: FSGS. Clin J Am Soc Nephrol 9: 626-632, 2014.

17. Nasr SH, Preddie DC, Markowitz GS, Appel GB and D'Agati VD: Multiple myeloma, nephrotic syndrome and crystalloid inclusions in podocytes. Kidney Int 69: 616-620, 2006.

18. Brenchley PE: Vascular permeability factors in steroid-sensitive nephrotic syndrome and focal segmental glomerulosclerosis. Nephrol Dial Transplant 18 (Suppl 6): vi21-vi25, 2003

19. Rajkumar SV and Kyle RA: Angiogenesis in multiple myeloma. Semin Oncol 28: 560-564, 2001.

20. Rajkumar SV, Mesa RA, Fonseca R, et al: Bone marrow angiogenesis in 400 patients with monoclonal gammopathy of undetermined significance, multiple myeloma, and primary amyloidosis. Clin Cancer Res 8: 2210-2216, 2002. 